\title{
Correlation of Emerging Substances and Physiological Groups of Microorganisms in Surface Water of River Moraca
}

\author{
Radonjic Drazana
}

Faculty of Science, Study group of Biology, George Washington Boulevard, Podgorica, Montenegro

\begin{abstract}
The article focuses on the reliable screening analyses of water quality of river Morača section near Podgorica, Montenegro. Sampling of screening analyses of surface water samples from the locality Vukovci, the lower course of the riverMorača during 2012 and 2013. The water samples were analysed by GC-MS. The compounds occurring most frequently in the analysed water samples were phthalates, PAHsdetergents, personal care products, flame retardants, , and corrosive residues, benzoate, pesticides, decane and the additive residues. Dibutyl phthalate, diethyl phthalate, dioctyl phthalate which are on the NORMAN list of emerging substances, and di(2ethylhexyl) phthalate, which is on the list of the WFD priority substances, were detected in all the examined samples. A large group of terpenes, such as nerol, citronellol, menthol, ionone, and compounds as camphor, ethyl citrate or methyl jasmonate that could be found in cosmetics, personal care products or home cleaning products were determined in river samples. The presence of hormones in all the surface water samples indicates human or animal faecal pollution, while the detected caffeine in all samples confirms an anthropogenic impact.A significant number of separated organic components spaces were not defined, which is acause for performance of microbiological analysis in the presence of physiological groups of microorganisms. The identified compounds can be associated with the presence of specific physiological groups of microorganisms at the site, which can in many ways reduce environmental stress due to their functional and significant role in ecosystem.
\end{abstract}

Keywords-water quality, emerging substances, the Morača, gas chromatography, physiological groups of microorganisms, environmental stress.

\section{INTRODUCTION}

In the natural aquatic environment diverse physical, chemical and biological processes occur and directly affect the content, transformation and movement of different constituents in water. A significant number of chemicals that can be found in water may have destructive impact on the environment and human health, often due to low level of knowledge and awareness, as well as lack of understanding of impacts and the toxicological implications [1].Generally, substances of concern tend to precipitate to sediment, which can represent a different level of problem, as sediment particles are often resistant to biodegradation, and most of all, have the high ability to bioaccumulate chemical substances. Emerging substances, present another level of concern, as low dose and pseudo-persistence can produce a very strong chemical and ecological stress in a long period of time, which can completely and irreversibly change the balance in the ecosystem as well as in the environment [2]. The importance of the low doses should be emphasized, especially for emerging substances (endocrine disruptive substances -EDCs), nano to pikogram (ppb toppt, respectively) concentrations, which mimic function and cycle of hormone like substances. For the purpose of emerging substances identification at the locality Vukovci $\left(42^{\circ} 27^{\prime} 81.5^{\prime}\right.$ ' $\mathrm{N}, 19^{\circ} 12^{\prime} 34.5^{\prime}$ ' $\left.\mathrm{E}\right)$ for the first time in the Republic of Montenegro, a screening study was conducted on 3 samples of River Morača surface water in 2 separate sampling campaigns.

Water samples from the locality were taken in November 2012. and in August of 2013. Analyses were performed on gas chromatographer coupled with mass spectrometer (GCMS), obtaining qualitative data about the chemical composition of samples, providing a range of substance groups varying from priority and hazardous, to emerging and benign. A screening analysis is a analytical process consisting of extraction, isolation and possible identification of a compound or group of compounds in a sample with the minimum number of steps and the minimal manipulation of the sample[3]. 
Suspected chemical that have been identified in surface water samples belong to emerging and priority groups of substances - detergents, personal care products, flame retardants, insecticides and pesticides, benzoate, pesticides, higher alkanes, additive residues were found. The screening analyses have shown a significant number of unidentified organic substances, which was the reason for microbiological analysis of water samples in the presence of physiological microorganism groups.

The surface water samples from the river Morača in locality Vukovci observed a significant presence of lipolytic bacteria: 4900 per $\mathrm{ml}$ of sample, and the presence of 20.000 colonies of proteolytic bacteria per ml of sample. These data show that the bacterial population is responsible for the transformation of the most organic micropolutants in environment. During the summer sampling a significantly smaller amount of physiological groups of microorganisms was determined - the amount of proteolytic bacteria was 130 bacterium per $\mathrm{ml}$ of sample, and lipolytic 17 bacteria per ml of sample.

\section{MATERIALSAND METHODS}

Location Vukovci is a part of sedimentation zone of the lower flow of River Morača, and in this part of the flow river has the characteristics of a typical lowland river. Surface water samples for screening and microbiological analyses have been collected simultaneously in two separate campaigns in November of 2012 and August of 2013. Samples have been taken from both sides of the River Morača. During the screening analyses several groups of priority and emerging substances have been identified.

Sampling was carried out at the location Vukovci, on both sides of the river. Sampling for microbiological and chemical analysis was carried out in the littoral segment of the river and sampled in pre-sterilized dark glass bottles.

Sampling bottles were washed and dried, then sterilized at $190{ }^{\circ} \mathrm{C}$ in a dry sterilizer for one hour. During the sampling, grab sampling procedure as prescribed by Water Act, 27/2007 of Montenegro was followed in full.A disposable sterile rubber stopper is carefully removed and the bottle is opened, with one hand holding the cap and the other hand grabbing the water sample, taking into account that the cap is not contaminated.

After sampling the bottle is tightly closed with a sterile cap. Sampling bottles for chemical analyses were rinsed with surface water three times before submerging for sampling, so the glass surface is chemically harmonized with sample. Sampling for microbiological analysis was done by quickly submerging prepared bottles, so the contamination of bottle is avoided. Samples were transported to the laboratory in portable fridge.

The screening analyses were performed on gas chromatograph Agilent $7890 \mathrm{~N}$ coupled with mass spectrometry detector Agilent 5975 at the Institute of Analytical Chemistry, Faculty of Chemical and Food Technology, Slovak University for Technology in Bratislava, Slovakia. Gas chromatography coupled with mass spectrometry analyses were performed on capillary column DB-FFAP $30 \mathrm{~m}$ x $250 \mathrm{~mm}$ I.D., $0.25 \mathrm{~mm}$, in scan acquisitionmode. Carrier gas was helium with flow 1 $\mathrm{ml} / \mathrm{min}$, oven program $40{ }^{\circ} \mathrm{C}, 10 \mathrm{~min}$ holding time; rate $2{ }^{\circ} \mathrm{C}$ $/$ min to $230{ }^{\circ} \mathrm{C}$, and splitlessinjector. Samples of surface water were prepared with liquid liquid extraction and evaporated in Kuderna Danish apparatus.

Liquid extraction was performed with different extraction solvents, polar and non-polar solvent, dichloromethane and pentane, respectively. Dichloromethane has shown to be a better choice for selected type of sample, in regard of efficiency and simplicity of liquid liquid extraction, as well as obtained chromatogram quality and mass fragments separation.

The microbiological analyses were performed in Hydrobiological Institute of Montenegro, Department of Biology. For the purpose of analyses the microbiological culture media were used and the ingredients for substrates used in this study are a product of the Institute for Immunology and Virology "Torlak" Belgrade, BioLiveMilano (Italy) and Seminem, Sarajevo (BiH). Substrates were prepared as specified by the manufacturer and sterilized in an autoclave for 15 to 20 minutes at $120^{\circ} \mathrm{C}$ under a pressure of 1.5 atmospheres.

\section{RESULTS AND DISCUSSION}

During the research conducted in the summer period gathered results for proteolyc and lipolytic bacteria showed lower number where proteolyc bacteria were represented in 130 bacteria per $\mathrm{ml}$ in a sample, and lipolytic bacteria 17 per $\mathrm{ml}$ in a sample. The obtained results are shown in Graphic 1. 


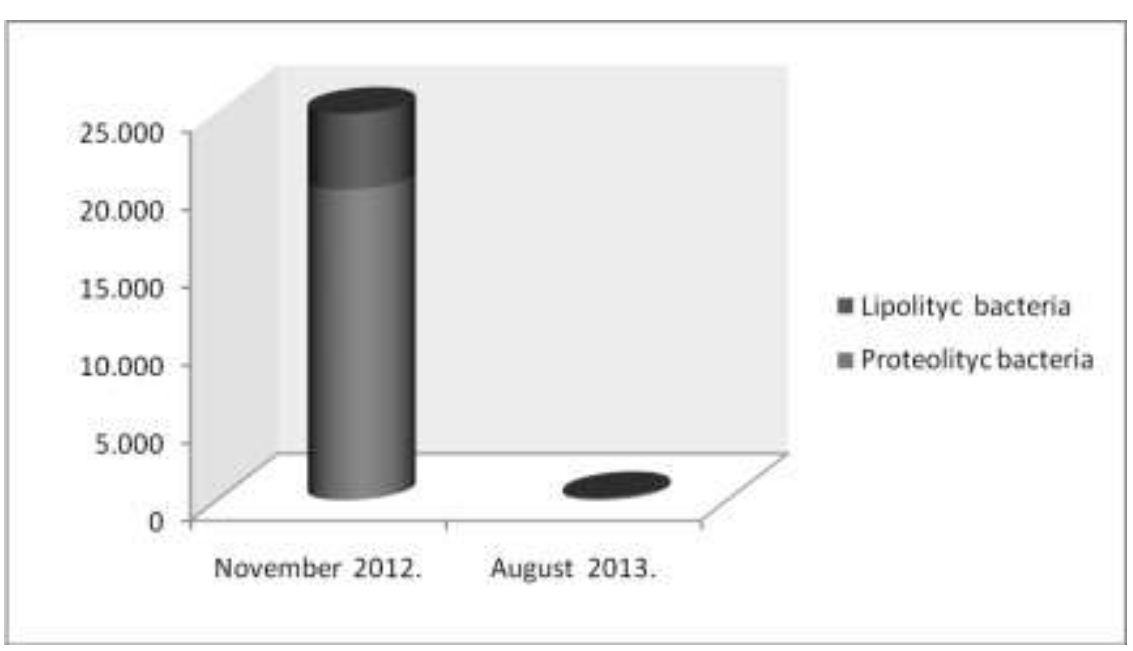

Graphic.1: Presence of proteolyc and lipolytic bacteria during winter and summer sampling

According to [4] the obtained results, due to the low level of water and high sludge thickness in the summer period, it can be concluded that the water condition results in overweight of coliform and bacteria of fecal origin, as well as intensive anaerobic process of organic substances decomposition in the sludge.

During the study in the November of 2012, significantly high content of bacteria was observed that the water samples from the river Moračanear locality Vukovci, lipolytic as well as proteolytic, 4,900 per ml of sample and 20,000 colonies of bacteria per $\mathrm{ml}$ of sample, respectively. Following study analysis conducted in Decemberthe presence of lipolytic and proteolytic bacteria was also detected. The amplitudes oflipolytic bacteria distribution was observed during seasonal changes, winter to summer period [5]during the year. Studying the quality of Čerava, the author draws attention on predominance of proteolytic bacteria compared to lipolytic. If literature data is compared to conducted research, the resemblances are evident.
The conclusion is that it could be a result of great amount of organic substances in water, which determines their distribution and development, meaning that we could assume that it depends on number of phytoplankton and macrophytes as well as organic alochtone nature. Proteolyc and lipolytic organisms are organisms performing the reduction and decomposition of a chemical compound to simpler forms, by utilizing the energy for their growth[6].Conducting the bacteriological analysis of the river Koselska water quality, it was determined that heterotrophic bacteria from every sample contained minimal qualities during the spring period, where maximal was determined in September, or late summer.

In November of 2012 during the first champagne 304 compounds have been detected, 183 of those were not identified. The identified substances with quality match index (QMI) higher than 60\% are shown in Table 1.

Table 1: Identified chemical components in water samples from the river Morače locality Vukovcianalyzed by GC-MS

\begin{tabular}{|r|l||c|c|c|}
\hline \multicolumn{1}{|c|}{ \# } & \multicolumn{1}{|c||}{ Compound name (CAS) } & QMI & Library & Samples \\
\hline \hline 1 & Benzene, methyl- & 94 & WILEY & 1 \\
\hline 2 & Disulfide, dimethyl & 95 & WILEY & 1 \\
\hline 3 & Cyclohexene, 1-methyl-4-(1-methylethenyl)- & 98 & WILEY & 1 \\
\hline 4 & 2-Oxabicyclo[2.2.2]octane, 1,3,3-trimethyl- & 80 & NIST & 1 \\
\hline 5 & 1-Butanol, 3-methyl- (impure) & 83 & WILEY & 1 \\
\hline 6 & 1-Pentanol & 61 & NIST & 1 \\
\hline 7 & 2,4-Dithiapentane & 76 & NIST & 1 \\
\hline 8 & 2-Pentanol, 4-methyl- & 80 & NIST & 1 \\
\hline 9 & Nonane, 1-chloro- & 60 & NIST & 1 \\
\hline 10 & Benzeneethanol
\end{tabular}




\begin{tabular}{|c|c|c|c|c|}
\hline 11 & Isopropyl myristate & 64 & NIST & 1 \\
\hline 12 & Hexadecanoic acid, methyl ester & 97 & WILEY & 1 \\
\hline 13 & Phenol, 2,6-dimethoxy- & 60 & NIST & 1 \\
\hline 14 & 9-Octadecenoic acid (Z)-, methyl ester & 97 & WILEY & 1 \\
\hline 15 & Octadecanoic acid & 91 & WILEY & 1 \\
\hline 16 & cis-9-Hexadecenoic acid & 67 & NIST & 1 \\
\hline 17 & Bicyclo[3.1.1]heptan-3-one, 2,6,6-trimethyl-, (1.alpha.,2.alpha.,5.alpha.)- & 64 & WILEY & 1 \\
\hline 18 & Myristoyl chloride & 60 & NIST & 1 \\
\hline 19 & Octadecanoic acid, 3-oxo-, methyl ester & 61 & NIST & 1 \\
\hline 20 & Methane, dichloro- & 64 & WILEY & 2 \\
\hline 21 & Pentane, 2,2-dimethyl- & 75 & NIST & 2 \\
\hline 22 & Acetic acid, (1,2-dimethyl-1-propenyl) ester & 65 & NIST & 2 \\
\hline 23 & Cyclohexane, octyl- & 70 & NIST & 2 \\
\hline 24 & Phytol & 62 & NIST & 2 \\
\hline 25 & Octadecane, 1-(ethenyloxy)- & 60 & NIST & 2 \\
\hline 26 & Cyclohexane, tetradecyl- & 64 & NIST & 2 \\
\hline 27 & Hexadecanoic acid & 99 & WILEY & 2 \\
\hline 28 & Hexadecenoic acid, Z-11-; & 78 & WILEY & 2 \\
\hline 29 & 9-Octadecenoic acid (Z)- & 97 & WILEY & 2 \\
\hline 30 & Octadecanoic acid, 2,3-dihydroxypropyl ester & 62 & NIST & 2 \\
\hline 31 & 2-Propanone, 1-hydroxy- & 72 & NIST & 3 \\
\hline 32 & 3-Furaldehyde & 69 & NIST & 3 \\
\hline 33 & 2-Furanmethanol & 75 & NIST & 3 \\
\hline 34 & 9-Octadecenoic acid & 99 & WILEY & 3 \\
\hline 35 & 9-Octadecenoic acid, (E)- & 89 & NIST & 3 \\
\hline 36 & 17-Octadecynoic acid & 66 & NIST & 3 \\
\hline 37 & Sulfurous acid, butyl pentadecyl ester & 66 & NIST & 4 \\
\hline 38 & Pentanal, 2,4-dimethyl- & 80 & NIST & 5 \\
\hline 39 & 3-Pentanol & 77 & NIST & 5 \\
\hline 40 & 1-Octanol, 2-butyl- & 70 & NIST & 6 \\
\hline 41 & n-Hexane & 71 & NIST & 1,2 \\
\hline 42 & 3-Ethyldibenzothiophene; & 78 & WILEY & 1,2 \\
\hline 43 & 2-Propanol, 1-hydrazino- & 64 & NIST & 1,3 \\
\hline 44 & 1-Chloroundecane & 64 & NIST & 1,3 \\
\hline 45 & 1-Tridecyne & 64 & NIST & 1,3 \\
\hline 46 & Tridecane, 6-cyclohexyl- & 69 & NIST & 1,4 \\
\hline 47 & Nonanal & & NIST & 1,5 \\
\hline 48 & 3-Hexanone, 2,5-dimethyl- & 68 & NIST & 1,6 \\
\hline 49 & 2-Hexanol, (S)- & 77 & NIST & 2,3 \\
\hline 50 & 1,3-Dioxan-4-one, 2-(1,1-dimethylethyl)-6-methyl- & 62 & NIST & 2,3 \\
\hline 51 & 2-Heptanol, acetate & 60 & NIST & 2,3 \\
\hline 52 & 3,3,5,5-Tetramethylcyclohexanol & 69 & NIST & 2,3 \\
\hline 53 & 4-Pyridinol-1-oxide & 60 & NIST & 2,3 \\
\hline 54 & 9,12-Octadecadienoic acid (Z,Z)- & 97 & WILEY & 2,3 \\
\hline 55 & Dihexylsulfide & 74 & WILEY & 2,4 \\
\hline 56 & Dodecane, 2,7,10-trimethyl- & 77 & NIST & 2,4 \\
\hline 57 & 17-Octadecynoic acid & 64 & NIST & 2,4 \\
\hline 58 & Bicyclo[4.1.0]heptane, 7-(1-methylethylidene)- & 81 & PBM & 2,5 \\
\hline
\end{tabular}




\begin{tabular}{|c|c|c|c|c|}
\hline 59 & Hexanedioic acid, dioctyl ester & 60 & NIST & 4,5 \\
\hline 60 & 1,6-Octadien-3-ol, 3,7-dimethyl- & 67 & NIST & 4,6 \\
\hline 61 & trans-3-Penten-2-ol & 78 & NIST & $1,2,3$ \\
\hline 62 & 2-Hexanol, 2,5-dimethyl-, (S)- & 67 & NIST & $1,2,3$ \\
\hline 63 & 1-Decanol & 66 & NIST & $1,2,3$ \\
\hline 64 & 2-Methyl-1-undecanol & 61 & NIST & $1,2,3$ \\
\hline 65 & Cyclodecane & 71 & NIST & $1,2,3$ \\
\hline 66 & Eicosane, 7-hexyl- & 61 & NIST & $1,2,4$ \\
\hline 67 & 9-Octadecenoic acid (Z)- & 99 & WILEY & $1,2,4$ \\
\hline 68 & Hexadecanoic acid & 99 & WILEY & $1,3,5$ \\
\hline 69 & Dodecane, 2,6,11-trimethyl- & 74 & NIST & $2,3,4$ \\
\hline 70 & 2-Hexyl-1-octanol & 66 & NIST & $2,3,4$ \\
\hline 71 & 2-Bromotetradecane & 65 & NIST & $2,3,4$ \\
\hline 72 & Octadecane, 3-methyl- & 66 & NIST & $2,3,4$ \\
\hline 73 & Nonadecane, 2-methyl- & 65 & NIST & $2,3,4$ \\
\hline 74 & n-Caproic acid vinyl ester & 75 & NIST & $2,3,6$ \\
\hline 75 & Allopregnane; Pregnane, (5.alpha.)- & 70 & WILEY & $2,4,5$ \\
\hline 76 & 1-Decanol, 2-octyl- & 60 & NIST & $2,4,6$ \\
\hline 77 & 2-Butanol, 3-methyl- & 74 & NIST & $4,5,6$ \\
\hline 78 & Eicosane, 2-methyl- & 73 & NIST & $1,3,4,5$ \\
\hline 79 & 7-Octen-1-ol, 3,7-dimethyl-, (S)- & 62 & NIST & $1,4,5,6$ \\
\hline 80 & 7,7-Diethylheptadecane & 69 & NIST & $2,3,5,6$ \\
\hline 81 & Trifluoroacetyl-lavandulol & 62 & NIST & $2,4,5,6$ \\
\hline 82 & 8-Azabicyclo[3.2.1]octan-3-amine, 8-methyl- & 62 & NIST & $3,4,5,6$ \\
\hline 83 & Hexadecane, 2,6,10,14-tetramethyl- & 73 & NIST & $1,2,3,4,6$ \\
\hline 84 & Cyclohexane, eicosyl- & 68 & NIST & $1,2,3,5,6$ \\
\hline 85 & 2,6-Octadien-1-ol, 3,7-dimethyl-, (Z)- & 72 & NIST & $1,3,4,5,6$ \\
\hline 86 & Oxalic acid, cyclohexylmethyltetradecyl ester & 61 & NIST & $1,3,4,5,6$ \\
\hline 87 & Tetracosane & 90 & WILEY & $1,3,4,5,6$ \\
\hline 88 & Decane, 2-methyl- & 81 & NIST & $2,3,4,5,6$ \\
\hline 89 & Cyclohexane, tetradecyl- & 64 & NIST & $2,3,4,5,6$ \\
\hline 90 & Sulfurous acid, butyl dodecyl ester & 71 & NIST & $2,3,4,5,6$ \\
\hline 91 & Undecane, 3-methyl- & 82 & NIST & $1,2,3,4,5,6$ \\
\hline 92 & 1-Octanol & 87 & NIST & $1,2,3,4,5,6$ \\
\hline 93 & Hexadecane & 86 & NIST & $1,2,3,4,5,6$ \\
\hline 94 & Heptadecane & 96 & WILEY & $1,2,3,4,5,6$ \\
\hline 95 & Dodecane, 2,6,10-trimethyl- & 79 & NIST & $1,2,3,4,5,6$ \\
\hline 96 & Octadecane & 97 & WILEY & $1,2,3,4,5,6$ \\
\hline 97 & Nonadecane & 91 & NIST & $1,2,3,4,5,6$ \\
\hline 98 & Eicosane & 93 & WILEY & $1,2,3,4,5,6$ \\
\hline 99 & Disulfide, di-tert-dodecyl & 71 & NIST & $1,2,3,4,5,6$ \\
\hline 100 & Eicosane, 3-methyl- & 70 & NIST & $1,2,3,4,5,6$ \\
\hline 101 & Hexadecane, 2,6,10,14-tetramethyl- & 72 & WILEY & $1,2,3,4,5,6$ \\
\hline 102 & Heneicosane & 95 & WILEY & $1,2,3,4,5,6$ \\
\hline 103 & 2,6-Diisopropylnaphthalene & 72 & NIST & $1,2,3,4,5,6$ \\
\hline 104 & 1-Tricosanol & 64 & NIST & $1,2,3,4,5,6$ \\
\hline 105 & Eicosane, 2,4-dimethyl- & 68 & NIST & $1,2,3,4,5,6$ \\
\hline 106 & Hydroxylamine, O-decyl- & 66 & NIST & $1,2,3,4,5,6$ \\
\hline
\end{tabular}




\begin{tabular}{|l|l||c|c|c|}
\hline 107 & Heneicosane, 3-methyl- & 66 & NIST & $1,2,3,4,5,6$ \\
\hline 108 & Docosane & 91 & WILEY & $1,2,3,4,5,6$ \\
\hline 109 & 1-Heneicosyl formate & 70 & NIST & $1,2,3,4,5,6$ \\
\hline 110 & 1-Tricosanol & 60 & NIST & $1,2,3,4,5,6$ \\
\hline 111 & Benzoic acid, 2-hydroxy-, phenylmethyl ester & 90 & WILEY & $1,2,3,4,5,6$ \\
\hline 112 & Tricosane, 2-methyl- & 75 & NIST & $1,2,3,4,5,6$ \\
\hline 113 & Tricosane & 81 & NIST & $1,2,3,4,5,6$ \\
\hline 114 & Heptadecane, 9-hexyl- & 61 & NIST & $1,2,3,4,5,6$ \\
\hline 115 & Cyclohexane, nonadecyl- & 78 & NIST & $1,2,3,4,5,6$ \\
\hline 116 & Heneicosane, 11-(1-ethylpropyl)- & 74 & NIST & $1,2,3,4,5,6$ \\
\hline 117 & Eicosane, 7-hexyl- & 69 & NIST & $1,2,3,4,5,6$ \\
\hline 118 & Octadecanoic acid & 99 & WILEY & $1,2,3,4,5,6$ \\
\hline 119 & Benzophenone & 81 & I.S. & $1,2,3,4,5,6$ \\
\hline 120 & Hexacosane & 76 & NIST & $1,2,3,4,5,6$ \\
\hline 121 & Octacosane & 91 & WILEY & $1,2,3,4,5,6$ \\
\hline 122 & Dibutyl phthalate & 85 & NIST & $1,2,3,4,5,6$ \\
\hline 123 & Diisooctyladipate & 71 & NIST & $1,2,3,4,5,6$ \\
\hline
\end{tabular}

More than $96 \%$ of the presented literature data from treated waste- and surface waters belong to high-income countries where industrial discharges are supposed to be controlled, e.g. Good Manufacturing Practices and emission regulations in the United States [7].In contrast, there is not enough data available from low- to middle-income countries where several manufacturing facilities are located and less strict regulations are applied.

During the summer research campaigned 63 compounds have been detected, 39 of those were not identified. The identified substances with quality match index (QMI) higher than $70 \%$ are shown in Table 2.Emerging Substances in the Aquatic Environment [8].can by Selected by based on Eco toxicological criteria. [9] Separated them on hidrophyleandlipophyle.

During the preparation of water ensamples we are using liquid extraction. [10] shoes to asimportance of organic chemicals in modern societies, pointing to their negative side. Summer screening, suggests significantly less presence of chemical substances, as well as chemical components that were unable to identify trough screening analysis.

Table 2: Identified chemical components in water samples from the river Morače locality Vukovcianalyzed by GC-MS

\begin{tabular}{|c|c|c|c|c|}
\hline $\begin{array}{l}\text { RT } \\
(\min )\end{array}$ & compound & $\begin{array}{l}\text { Quality } \\
\text { match }\end{array}$ & Notes & Samples \\
\hline 90.331 & Hexadecanoic acid (CAS); Palmitic acid & 99 & WILEY & 2 \\
\hline 96.817 & 9-Octadecenoic acid (Z)- (CAS); Oleic acid; & 99 & WILEY & 3 \\
\hline 99.507 & 9-Octadecenoic acid (Z)- (CAS); Oleic acid; & 99 & WILEY & $1,2,4$ \\
\hline 92.81 & Hexadecanoic acid (CAS); Palmitic acid; & 99 & WILEY & $1,3,5$ \\
\hline 91.167 & Octadecanoic acid & 99 & WILEY & $1,2,3,4,5,6$ \\
\hline 22.613 & $\begin{array}{l}\text { dl-Limonene; Cyclohexene, 1-methyl-4-(1- } \\
\text { methylethenyl)- }\end{array}$ & 98 & WILEY & 1 \\
\hline 81.201 & $\begin{array}{l}\text { Hexadecanoic acid, methyl ester (CAS); Methyl } \\
\text { palmitate }\end{array}$ & 97 & WILEY & 1 \\
\hline 90.911 & $\begin{array}{l}\text { 9-Octadecenoic acid (Z)-, methyl ester (CAS); Methyl } \\
\text { oleate; }\end{array}$ & 97 & WILEY & 1 \\
\hline 97.66 & Oleic Acid; 9-Octadecenoic acid (Z)- & 97 & WILEY & 2 \\
\hline 102.855 & 9,12-Octadecadienoic acid (Z,Z)- (CAS); Linoleic & 97 & WILEY & 2,3 \\
\hline
\end{tabular}




\begin{tabular}{|c|c|c|c|c|}
\hline & acid; & & & \\
\hline 14.369 & Disulfide, dimethyl & 95 & WILEY & 1 \\
\hline 95.673 & Octadecanoic acid (CAS); Stearic acid; & 91 & WILEY & 1 \\
\hline 83.963 & $\begin{array}{l}\text { Benzoic acid, 2-hydroxy-, phenylmethyl ester (CAS); } \\
\text { Benzyl salicylate; }\end{array}$ & 90 & WILEY & $1,2,3,4,5,6$ \\
\hline 97.129 & 9-Octadecenoic acid, (E)- & 89 & NIST & 3 \\
\hline 46.969 & 1-Octanol & 87 & NIST & $1,2,3,4,5,6$ \\
\hline 100.73 & Dibutyl phthalate & 85 & NIST & $1,2,3,4,5,6$ \\
\hline 26.647 & 1-Pentanol (CAS); Amylol & 83 & WILEY & 1 \\
\hline 44.716 & Undecane, 3-methyl- & 82 & NIST & $1,2,3,4,5,6$ \\
\hline 22.607 & Bicyclo[4.1.0]heptane, 7-(1-methylethylidene)-; & 81 & PBM & 2,5 \\
\hline 38.266 & Decane, 2-methyl- & 81 & NIST & $2,3,4,5,6$ \\
\hline 23.548 & 1-Butanol, 3-methyl- (impure) & 80 & WILEY & 1 \\
\hline 53.488 & Nonane, 1-chloro- & 80 & NIST & 1 \\
\hline 60.926 & Dodecane, 2,6,10-trimethyl- & 79 & NIST & $1,2,3,4,5,6$ \\
\hline 93.869 & Hexadecenoic acid, Z-11-; & 78 & WILEY & 2 \\
\hline 81.9 & 3-Ethyldibenzothiophene; & 78 & WILEY & 1,2 \\
\hline 20.915 & trans-3-Penten-2-ol & 78 & NIST & $1,2,3$ \\
\hline 87.389 & Cyclohexane, nonadecyl- & 78 & NIST & $1,2,3,4,5,6$ \\
\hline 30.065 & 2-Pentanol, 4-methyl- & 76 & NIST & 1 \\
\hline 53.331 & 2-Furanmethanol & 75 & NIST & 3 \\
\hline 43.798 & n-Caproic acid vinyl ester & 75 & NIST & $2,3,6$ \\
\hline 41.142 & dihexylsulfide & 74 & WILEY & 2,4 \\
\hline 17.101 & 2-Butanol, 3-methyl- & 74 & NIST & $4,5,6$ \\
\hline 88.203 & Heneicosane, 11-(1-ethylpropyl)- & 74 & NIST & $1,2,3,4,5,6$ \\
\hline 30.264 & 2-Propanone, 1-hydroxy- & 72 & NIST & 3 \\
\hline 60.735 & 2,6-Octadien-1-ol, 3,7-dimethyl-, (Z)- ; (Nerol) & 72 & & $1,3,4,5,6$ \\
\hline 78.332 & 2,6-Diisopropylnaphthalene & 72 & NIST & $1,2,3,4,5,6$ \\
\hline 101.884 & Sulfurous acid, butyl dodecyl ester & 71 & NIST & $2,3,4,5,6$ \\
\hline 73.997 & Disulfide, di-tert-dodecyl & 71 & NIST & $1,2,3,4,5,6$ \\
\hline 104.383 & Diisooctyladipate & 71 & NIST & $1,2,3,4,5,6$ \\
\hline 50.169 & Cyclohexane, octyl- & 70 & NIST & 2 \\
\hline 74.823 & 1-Octanol, 2-butyl- & 70 & NIST & 6 \\
\hline 93.365 & Allopregnane; Pregnane, (5.alpha.)- & 70 & WILEY & $2,4,5$ \\
\hline 82.792 & 1-Heneicosyl formate & 70 & NIST & $1,2,3,4,5,6$ \\
\hline 41.669 & 3-Furaldehyde & 69 & NIST & 3 \\
\hline 82.815 & Tridecane, 6-cyclohexyl- & 69 & NIST & 1,4 \\
\hline 54.069 & 3,3,5,5-Tetramethylcyclohexanol & 69 & NIST & 2,3 \\
\hline 81.116 & 7,7-Diethylheptadecane & 69 & NIST & $2,3,5,6$ \\
\hline 23.043 & $\begin{array}{l}\text { 2-Oxabicyclo[2.2.2]octane, 1,3,3-trimethyl- } \\
\text {;Eucalyptol }\end{array}$ & 67 & NIST & 1 \\
\hline 46.421 & 1,6-Octadien-3-ol, 3,7-dimethyl- & 67 & NIST & 4,6 \\
\hline
\end{tabular}




\begin{tabular}{|c|c|c|c|c|}
\hline 103.74 & 17-Octadecynoic acid & 66 & NIST & 3 \\
\hline 96.316 & Sulfurous acid, butyl pentadecyl ester & 66 & NIST & 4 \\
\hline 58.105 & 1-Decanol & 66 & NIST & $1,2,3$ \\
\hline 65.481 & Octadecane, 3-methyl- & 66 & NIST & $2,3,4$ \\
\hline 79.368 & Hydroxylamine, O-decyl- & 66 & NIST & $1,2,3,4,5,6$ \\
\hline 31.664 & Acetic acid, (1,2-dimethyl-1-propenyl) ester & 65 & NIST & 2 \\
\hline 65.136 & 2-Bromotetradecane & 65 & NIST & $2,3,4$ \\
\hline 72.967 & Isopropyl myristate & 64 & NIST & 1 \\
\hline 97.762 & $\begin{array}{l}\text { Bicyclo[3.1.1]heptan-3-one, 2,6,6-trimethyl-, } \\
\text { (1.alpha.,2.alpha.,5.alpha.)-; Pinocamphone; }\end{array}$ & 64 & WILEY & 1 \\
\hline 13.09 & $\begin{array}{l}\text { Methane, dichloro- (CAS); Dichloromethane; R 30; } \\
\text { Freon 30; Narkotil; }\end{array}$ & 64 & WILEY & 2 \\
\hline 72.921 & Cyclohexane, tetradecyl- & 64 & NIST & 2 \\
\hline 49.205 & 2-Propanol, 1-hydrazino- & 64 & NIST & 1,3 \\
\hline 59.371 & 1-Chloroundecane & 64 & NIST & 1,3 \\
\hline 61.381 & 1-Tridecyne & 64 & NIST & 1,3 \\
\hline 104.062 & 17-Octadecynoic acid & 64 & NIST & 2,4 \\
\hline 78.66 & 1-Tricosanol & 64 & NIST & $1,2,3,4,5,6$ \\
\hline 68.95 & Phytol & 62 & NIST & 2 \\
\hline 103.822 & Octadecanoic acid, 2,3-dihydroxypropyl ester & 62 & NIST & 2 \\
\hline 45.182 & 1,3-Dioxan-4-one, 2-(1,1-dimethylethyl)-6-methyl- & 62 & NIST & 2,3 \\
\hline 58.919 & 7-Octen-1-ol, 3,7-dimethyl-, (S)- & 62 & & $1,4,5,6$ \\
\hline 63.287 & Trifluoroacetyl-lavandulol & 62 & NIST & $2,4,5,6$ \\
\hline 72.951 & 8-Azabicyclo[3.2.1]octan-3-amine, 8-methyl- & 62 & NIST & $3,4,5,6$ \\
\hline 28.949 & 2,4-Dithiapentane; Formaldehyde dimethyl mercaptal & 61 & NIST & 1 \\
\hline 104.534 & Octadecanoic acid, 3-oxo-, methyl ester & 61 & NIST & 1 \\
\hline 59.669 & 2-Methyl-1-undecanol & 61 & NIST & $1,2,3$ \\
\hline 85.038 & Oxalic acid, cyclohexylmethyltetradecyl ester & 61 & NIST & $1,3,4,5,6$ \\
\hline 66.51 & Benzeneethanol (CAS); Phenethyl alcohol & 60 & NIST & 1 \\
\hline 83.477 & Phenol, 2,6-dimethoxy- & 60 & NIST & 1 \\
\hline 98.516 & Myristoyl chloride & 60 & NIST & 1 \\
\hline 72.528 & Octadecane, 1-(ethenyloxy)- & 60 & NIST & 2 \\
\hline 45.569 & 2-Heptanol, acetate & 60 & NIST & 2,3 \\
\hline 56.085 & 4-Pyridinol-1-oxide & 60 & NIST & 2,3 \\
\hline 103.78 & Hexanedioic acid, dioctyl ester & 60 & NIST & 4,5 \\
\hline 92.915 & 1-Decanol, 2-octyl- & 60 & NIST & $2,4,6$ \\
\hline 83.162 & 1-Tricosanol & 60 & NIST & $1,2,3,4,5,6$ \\
\hline
\end{tabular}



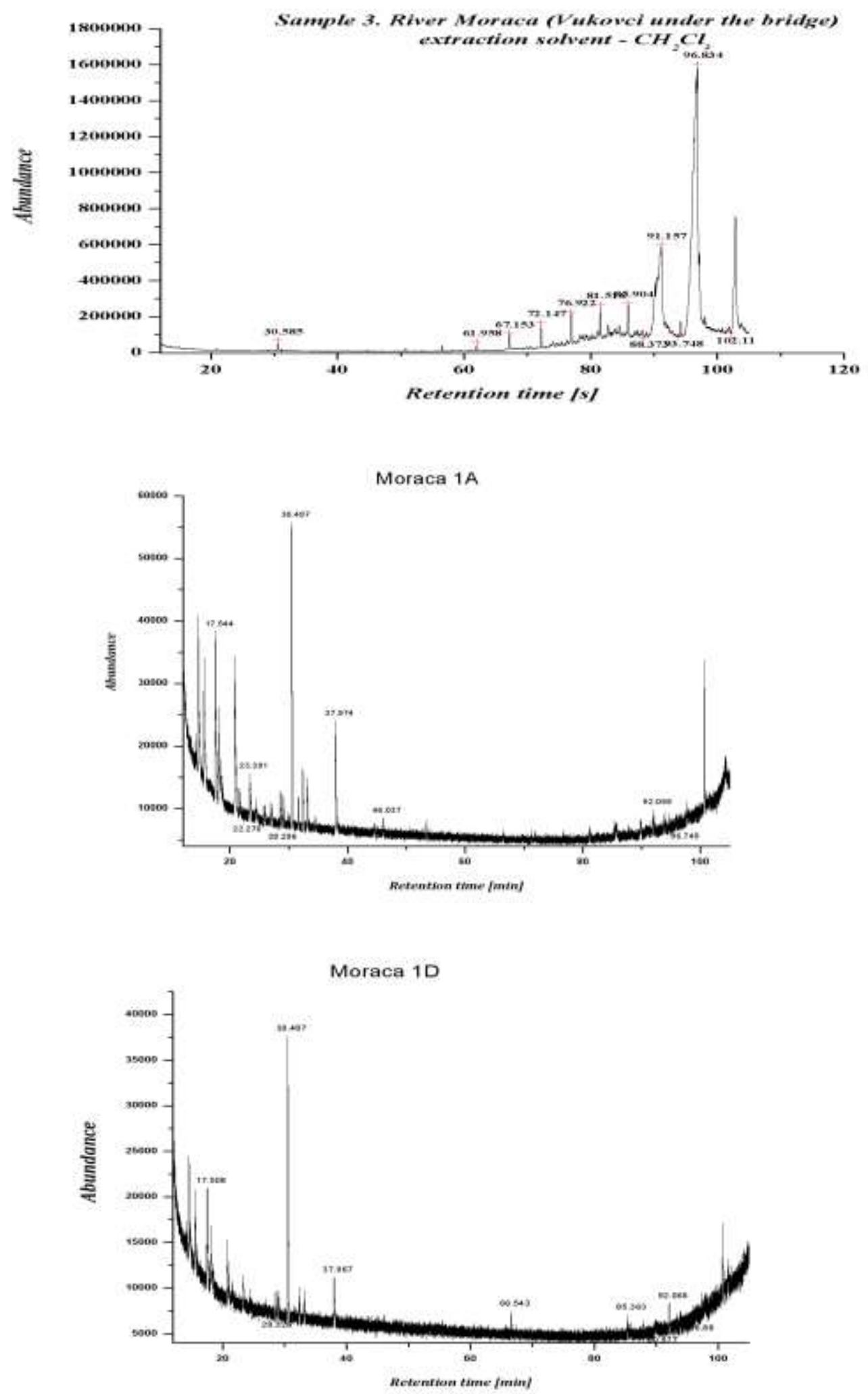
The screening analysis of water samples is indicating the present of certain emerging substances: phenolic and benzene derivates, detergents, personal care products, irritants, benzoate, pesticides, isohexadecane, Flammable substances and residues corrosives.

The reduction of emerging species is evident during August of 2013. Compared to the November of 2012. This can certainly be explained by the difference in atmospheric and water temperature, the significant reduction of river flows divergence of aquatic life and etc. During the summer and winter period the river Morača on the site of Vukovci is significantly different, by water flow and volume, which is certainly reflected onto the water quality and present of aquatic life, which can be observed in Picture 1.

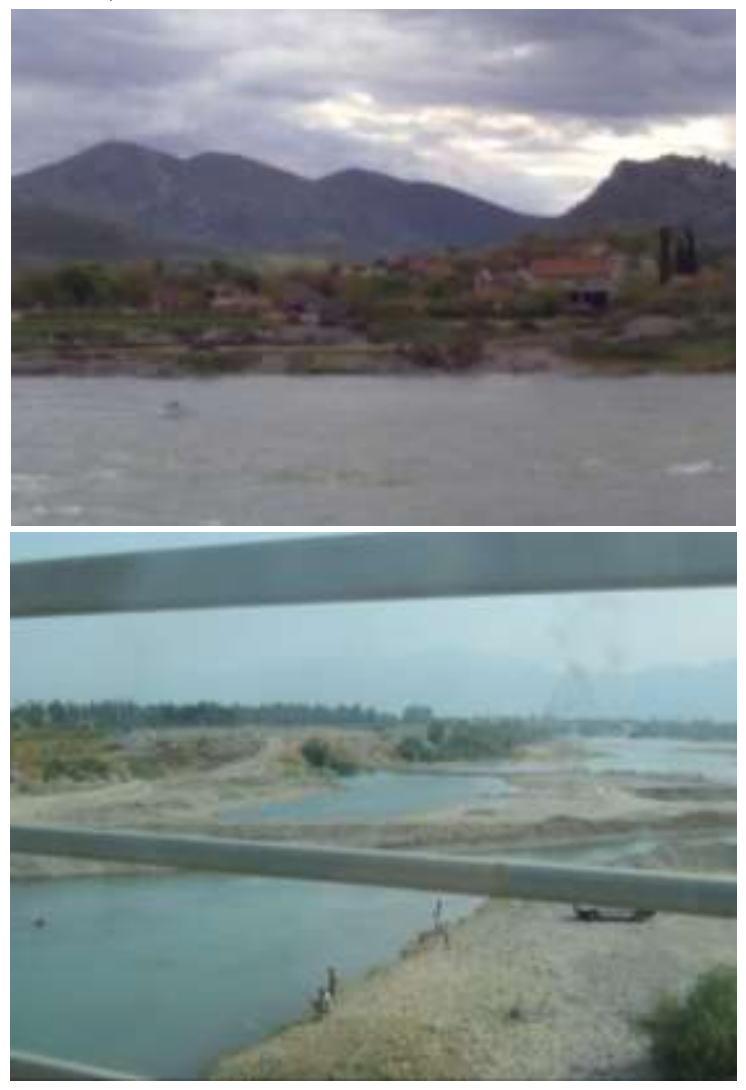

Picture.1: Locality Vukovci, taken by author during the winter and summer sampling

The persistence of the chemicals identified as emerging substances, during the screening analysis conducted in the August of 2013, indicates the consistent input of certain chemicals in surface, their persistency and potency for deposition in ecosystem, and, if necessary, reactivation during optimal period.

In literature source [12]it is emphasised that the fate and content of pharmaceuticals and other emerging substances in surface and ground water can be associated with the content of coliform bacteria in water. The presence of bacteria in water shows evidence of organic influence on water quality[13].Microorganisms have the potency for adapting to new conditions and existing organic pollutants due to the relevant mutations that will spread through the population. The process is known as adaptation, characterized by longer and less reproducible initial period, before degradation can be observed. After the adaptation period the aquatic population of specific location will be able to breaks down a substance without the lengthy initial phase [14].

\section{CONCLUSIONS}

The presence of physiological groups of microorganisms can be a significant indicator of organic pollution in surface water caused by chemical substances introduced into the water body from various sources. The identification of physiological groups of microorganisms in the study of locality Vukovci certainly can be correlated with the presence of emerging substances or their transformation metabolites in water.

The surface watersensitive to natural and antropogenic impacts occuring daily, which can accelerate, decelerate or pospone the transformation processes - (bio)degradation, adsorption, absorption, photolysis, hydrolysis, oxidation/reduction and etc.The significance of these processes is reflected in normal functioning of an ecosystem, natural river ecosystem. Every chaneg of chemical content in aquatic system is causing the corresponding reaction. The microorganisms are adapting to changes so the impact on natural ecosystem can be neutralized. The toxicity, persistancy and biodegradation properties of chemical entities introduced to the ecosystem (naturaly or antropogenicaly) have the most important influence onto the microorganisms and their ability to adapt to changed conditions.

Microorganisms and their activity can be a crucial indicator for a change or instability of an aquatic ecosystem as well as a powerfull mechanism of its recovery. Taking into account that the microorganisms are the best natural source of remediation, we can conclude that their presence is constant with the presence of emerging substances in selected location Vukovci. 


\section{REFERENCES}

[1] Đukić, D., Jermecev, B. T. (2003): Mikrobiološkabiotehnologija."Deret" Belgrade,Vol. 503. pg.25-27

[2] Miloradov - Vojinović, M., Miloradov, M.,SekulićTurk, M., Radonić, J., Stošić, M., (2012): Low doses effects of Emerging substances, pseudopersistancy and hazard concequences to aquatic enviromentt and public heslth,2. International Conference "Ecology of urban areas 2012", Zrenjanin, 2012

[3] Mun oz-Olivas,R. (2004) Trends in Analytical Chemistry, Vol. 23, No. 3, 203-2016, Elsevier B.V. doi:10.1016/S0165-9936(04)00318-8 [4] Radonjić,
Utvrđivanjekvalitetavodenanekimlokacijama

Skadarskogjezerai Rijeka Moračei BojaneMagistarski rad

[5] Lokovska, L. (2003a): Bakteriološkaanalizarijeke Čerave, 32. Konferencija o aktuelnim problemimakorišćenja I zaštitevoda, Voda 2003. Zlatibor, 2003. pg. 305-309

[6] Lokovska, L. (2003b): BakteriološkakontrolakvalitetavodeKoselskereke, 32. Konferencija 0 aktuelnimproblemimakorišćenja I zaštitevoda, Voda 2003. Zlatibor 2003. pg. 309-314.

[7] Velagaleti, R., Burns, P.K., Gill, M. \&Prothro, J. (2002). Impact of current good manufacturing practices and emission regulations and guidances on the discharge of pharmaceutical chemicals into the environment from manufacturing, use, and disposal. Environmental Health Perspectives 110(3), pg. 213220

[8] Abdel-El-Haleem, D. (2003) Acinetobacter: environmental and biotechnological applications. African Journal of Biotechnology Vol. 2(4), pg. 7174

[9] Ternes TA (2006). Consumption and Occurrence. In Human Pharmaceuticals, Hormones and Fragrances: The challenge of micro pollutants in urban water management , Ternes TA, Joss A (Eds.). IWA Publishing, London, UK, pp. 15-54

[10] Richardson S. D. Water analysis: emerging contaminants and current issues. Analytical Chemistry. 2007;79(12):4295-4323. doi: 10.1021/ac070719q. [PubMed] [Cross Ref]

[11] Al-Ahmad, A., Al-Ahmad,MW.,Schon, G., Daschner, F.D., Kummerer, K. (2000) Role of Acinetobacter for biodegradability of quaternary ammonium compounds. Bulletin of Environmental Contamination and Toxicology Vol. 64 pg. 764-770

[12] Daneshvar,A. (2012): Source, Occurrence, and Fate of Pharmaceuticals in Natural Waters-Doctoral Thesis

[13] Petrovic, O., Knežević, P., Simeunović, J., Rončević, S., Ivanović, D. (2009): IzvorišteŠtrandmikrobiološkikvalitetpodzemnevode sanitarnazaštita; Vodaisanitarnatehnika, 2009, Vol. 39 (5) pg. 9-14

[14] Abrahamsson, K., Klick, S. (1991) Degradation of halogenated phenols inanoxic natural marine sediments. Marine Pollution. BulletinVol. 22 pg. 227-233. 